\title{
Table algebras with multiple P-polynomial structures
}

\author{
Bangteng Xu
}

Received: April 21, 2005 / Revised: October 12, 2005 / Accepted: October 19, 2005

(C) Springer Science + Business Media, LLC 2006

\begin{abstract}
Using covering numbers we prove that a standard real integral table algebra $(A, \mathbf{B})$ with $|\mathbf{B}| \geq 6$ has a P-polynomial structure with respect to every $b \neq 1$ in $\mathbf{B}$ if and only if $2|\mathbf{B}|-1$ is prime and $(A, \mathbf{B})$ is exactly isomorphic to the Bose-Mesner algebra of the association scheme of the ordinary $(2|\mathbf{B}|-1)$-gon. Then we present an example showing that this result is not true if $|\mathbf{B}| \leq 5$.
\end{abstract}

Keywords Table algebras · Covering numbers $\cdot$ Association schemes $\cdot$ Bose-Mesner algebras · P-polynomial structures

\section{Introduction}

C-algebras of P-polynomial type were first studied by Bannai and Ito [4]. A C-algebra ( $A, \mathbf{B})$ is of $\mathrm{P}$-polynomial type if and only if there is a $b \neq 1$ in $\mathbf{B}$ such that the intersection matrix of $b$ with respect to $\mathbf{B}$ or a reordering of $\mathbf{B}$ is tridiagonal of the form

$$
\left(\begin{array}{cccccc}
0 & 1 & & & & \\
\alpha_{0} & \beta_{1} & \gamma_{2} & & & \\
& \alpha_{1} & \beta_{2} & \gamma_{3} & & \\
& & \ddots & \ddots & \ddots & \\
& & & \alpha_{k-2} & \beta_{k-1} & \gamma_{k} \\
& & & & \alpha_{k-1} & \beta_{k}
\end{array}\right), \quad \text { where } \alpha_{i}>0, \gamma_{j}>0
$$

C-algebras of P-polynomial type are important because an association scheme has a $\mathrm{P}$-polynomial structure if and only if its Bose-Mesner algebra is a C-algebra of P-polynomial type. It is well-known that a symmetric association scheme which is not derived from a polygon can only have at most two P-polynomial structures, while the association scheme

B. Xu (ه)

Department of Mathematics and Statistics, Eastern Kentucky University, Richmond, KY 40475 e-mail: bangteng.xu@eku.edu 
of an ordinary $n$-gon with $n$ prime has $(n-1) / 2$ P-polynomial structures (see [4, Theorem 4.2 , p. 241]). That is, if $(A, \mathbf{B})$ is the Bose-Mesner algebra of the association scheme of an ordinary $n$-gon with $n$ prime, then for any $b \neq 1$ in $\mathbf{B}$, the intersection matrix of $b$ with respect to $\mathbf{B}$ or some reordering of $\mathbf{B}$ is tridiagonal of the form (1). Now suppose $(A, \mathbf{B})$ is a $\mathbf{C}$-algebra with nonnegative integral structure constants such that for any $b \neq 1$ in $\mathbf{B}$, the intersection matrix of $b$ with respect to $\mathbf{B}$ or some reordering of $\mathbf{B}$ is tridiagonal of the form (1). Is $(A, \mathbf{B})$ exactly isomorphic to the Bose-Mesner algebra of the association scheme of some ordinary $n$-gon with $n$ prime?

Table algebras are C-algebras with nonnegative structure constants. Arad and Blau [1] introduced the concept of the covering number $\operatorname{cn}(\mathbf{B})$ of a table algebra $(A, \mathbf{B})$, and proved that $c n(\mathbf{B})$ exists if and only if $(A, \mathbf{B})$ is simple and nonabelian, and that $\operatorname{cn}(\mathbf{B}) \leq\left(|\mathbf{B}|^{2}-\right.$ $\left.(r-1)^{2}\right) / 2$ if $c n(\mathbf{B})$ exists, where $r$ is the number of real $b_{i} \neq 1$ in $\mathbf{B}$. In particular, if $(A, \mathbf{B})$ is a real table algebra and $\operatorname{cn}(\mathbf{B})$ exists, then $\operatorname{cn}(\mathbf{B}) \leq 2|\mathbf{B}|-2$. Arad and Blau [2] constructed an infinite family of real table algebras $(A, \mathbf{B})$ such that $\operatorname{cn}(\mathbf{B})$ exists and $\operatorname{cn}(\mathbf{B})=2|\mathbf{B}|-2$. The first intersection matrices of these table algebras are tridiagonal of the form

$$
\left(\begin{array}{cccccc}
0 & 1 & & & & \\
\alpha_{0} & 0 & \gamma_{2} & & & \\
& \alpha_{1} & 0 & \gamma_{3} & & \\
& & \ddots & \ddots & \ddots & \\
& & & \alpha_{k-2} & 0 & \gamma_{k} \\
& & & & \alpha_{k-1} & \lambda_{k}
\end{array}\right) \text {, where } \alpha_{i}>0, \gamma_{j}>0, \lambda_{k}>0
$$

However, for a real table algebra $(A, \mathbf{B})$ whose first intersection matrix is of the form (2), $\operatorname{cn}(\mathbf{B})$ may not exist (see Example 1.2 below).

In this paper we will answer the question proposed at the end of the first paragraph by the use of covering numbers (see Theorem 3.4). We will first generalize the concept of the covering number of a table algebra $(A, \mathbf{B})$. For any $b \in \mathbf{B}$, we define the covering number $c n(b)$ of $b$ (Definition 2.1), and provide a necessary and sufficient condition under which $c n(b)$ exists (Proposition 2.2), as well as an upper bound of $c n(b)$ when $c n(b)$ exists (Proposition 2.3). Our results generalize the results of Arad and Blau [1, Theorem B]. Then, using the covering number of an element $b \in \mathbf{B}$, we present a characterization for a table algebra $(A$, B) such that the first intersection matrix is of the form (2). We will prove that, by a suitable renumbering of $b_{i} \in \mathbf{B}$ if necessary, the first intersection matrix of $(A, \mathbf{B})$ is of the form (2) if and only if there is a $b \in \mathbf{B}$ such that $c n(b)$ exists and $c n(b)=2|\mathbf{B}|-2$ (Theorem 2.5). For a real table algebra $(A, \mathbf{B})$, we will present necessary and sufficient conditions under which for any $b \neq 1$ in $\mathbf{B}, c n(b)$ exists and $c n(b)=2|\mathbf{B}|-2$ (Proposition 3.2 and Theorem 3.3). Finally, using these results we prove that a standard real integral table algebra $(A, \mathbf{B})$ with $|\mathbf{B}| \geq 6$ is exactly isomorphic to the Bose-Mesner algebra of the association scheme of some ordinary $n$-gon with $n$ prime if and only if for any $b \neq 1$ in $\mathbf{B}$, the intersection matrix of $b$ with respect to $\mathbf{B}$ or some reordering of $\mathbf{B}$ is tridiagonal of the form (1) (Theorem 3.4).

The rest of this introductory section gives notation, definitions, and examples. Throughout this paper, $\mathbb{C}$ denotes the complex numbers, $\mathbb{R}^{+}$the positive real numbers, and $\mathbb{N}$ the positive integers.

Definition 1.1. Let $\mathbf{B}=\left\{b_{0}, b_{1}, \ldots, b_{k}\right\}$ be a basis of a finite dimensional, associative, and commutative algebra $\mathrm{A}$ over $\mathbb{C}$ such that $b_{0}=1$, the identity element of $\mathrm{A}$. $(A, \mathbf{B})$ is called a table algebra and $\mathbf{B}$ a table basis of $\mathrm{A}$ if the following hold. 
(i) For all $i, j, m, b_{i} b_{j}=\sum_{m=0}^{k} \beta_{i j m} b_{m}$ with $\beta_{i j m} \in \mathbb{R}^{+} \cup\{0\}$;

(ii) There is an algebra automorphism (denoted by ${ }^{-}$) of A whose order divides 2 , such that $b_{i} \in \mathbf{B}$ implies that $\bar{b}_{i} \in \mathbf{B}$ (then $\bar{i}$ is defined by $b_{\bar{i}}=\bar{b}_{i}$ );

(iii) For all $i, j, \beta_{i j 0} \neq 0 \Leftrightarrow j=\bar{i}$; and $\beta_{i \bar{i} 0}>0$.

Let $(A, \mathbf{B})$ be a table algebra with $\mathbf{B}=\left\{b_{0}, b_{1}, \ldots, b_{k}\right\}$. Then there is a unique algebra homomorphism $f: A \rightarrow \mathbb{C}$ such that $f\left(b_{i}\right)=f\left(\bar{b}_{i}\right)>0$ for all $b_{i} \in \mathbf{B}$. The number $f\left(b_{i}\right)$ is called the degree of $b_{i}$. If $f\left(b_{i}\right)=\beta_{i \bar{i} 0}$ for all $b_{i} \in \mathbf{B}$, then $(A, \mathbf{B})$ is called a standard table algebra. For any table algebra $(A, \mathbf{B})$, there is a rescaling $\mathbf{B}^{\prime}$ of $\mathbf{B}$ such that $\left(\mathrm{A}, \mathbf{B}^{\prime}\right)$ is a standard table algebra. A C-algebra with nonnegative structure constants in the sense of $[4$, p. 88$]$ is a standard table algebra. A table algebra $(A, \mathbf{B})$ is called an integral table algebra if all the structure constants $\beta_{i j m}$ and all the degrees $f\left(b_{i}\right)$ are integers.

Let $(A, \mathbf{B})$ be a table algebra and $b_{i} \in \mathbf{B}$. If $\bar{b}_{i}=b_{i}$, then $b_{i}$ is called real. If every $b_{i} \in \mathbf{B}$ is real, then $\mathbf{B}$ is called real and $(A, \mathbf{B})$ is called a real table algebra.

Let $(A, \mathbf{B})$ be a table algebra with $\mathbf{B}=\left\{b_{0}=1, b_{1}, \ldots, b_{k}\right\}$. Let's regard $\mathbf{B}$ as a linearly ordered set. Let $\sigma$ be a permutation of $\{0,1,2, \ldots, k\}$ with $\sigma(0)=0$. Then the ordered set $\sigma(\mathbf{B}):=\left\{b_{0}, b_{\sigma(1)}, \ldots, b_{\sigma(k)}\right\}$ is called a reordering of $\mathbf{B}$. For any $b_{i} \in \mathbf{B}$, there is a unique $(k+1) \times(k+1)$ matrix

$$
B_{i}=\left(\begin{array}{cccc}
\beta_{i 00} & \beta_{i 01} & \cdots & \beta_{i 0 k} \\
\beta_{i 10} & \beta_{i 11} & \cdots & \beta_{i 1 k} \\
\vdots & \vdots & \ddots & \vdots \\
\beta_{i k 0} & \beta_{i k 1} & \cdots & \beta_{i k k}
\end{array}\right)
$$

with nonnegative real entries such that

$$
b_{i} b_{\sigma(j)}=\beta_{i j 0} b_{0}+\beta_{i j 1} b_{\sigma(1)}+\cdots+\beta_{i j k} b_{\sigma(k)}, \quad j=0,1,2, \ldots, k .
$$

The matrix $B_{i}$ is called the intersection matrix of $b_{i}$ with respect to the ordered basis $\sigma(\mathbf{B})$, and denoted by $\operatorname{Mat}\left(b_{i}\right)_{\sigma(\mathbf{B})}$. Throughout this paper, we always regard the basis $\mathbf{B}$ and its reordering $\sigma(\mathbf{B})$ as ordered sets whenever intersection matrices are involved. Usually $\operatorname{Mat}\left(b_{1}\right)_{\mathbf{B}}$ is called the first intersection matrix of $(A, \mathbf{B})$.

Definition 1.2. Let $(A, \mathbf{B})$ be a real table algebra and $b_{i} \in \mathbf{B}$. If there is a reordering $\mathbf{B}^{\prime}$ of $\mathbf{B}$ such that $\operatorname{Mat}\left(b_{i}\right)_{\mathbf{B}^{\prime}}$ is tridiagonal of the form (1), then we say that $(A, \mathbf{B})$ has a P-polynomial structure with respect to $b_{i}$. In this case $(A, \mathbf{B})$ is also called a table algebra of P-polynomial type.

Let $(A, \mathbf{B})$ be a table algebra and $\mathbf{B}=\left\{b_{0}, b_{1}, \ldots, b_{k}\right\}$. For any $\alpha \in \mathbb{C}$, let $\alpha^{*}$ be the complex conjugate of $\alpha$. For any $x, y \in A, x=\sum_{i=0}^{k} \alpha_{i} b_{i}$ and $y=\sum_{i=0}^{k} \gamma_{i} b_{i}$ for unique $\alpha_{i}, \gamma_{i} \in \mathbb{C}$, define

$$
(x, y)=\sum_{i=0}^{k} \beta_{i \bar{i} 0} \alpha_{i} \gamma_{i}^{*} \quad \text { and } \quad x^{*}=\sum_{i=0}^{k} \alpha_{i}^{*} b_{i} .
$$


Then (, ) is a positive definite Hermitian form of A and has $\mathbf{B}$ as an orthogonal basis. Furthermore, for any $x, y, z \in A,(x y, z)=\left(x, \bar{y}^{*} z\right)$. In particular, for any $b_{i}, b_{j}, b_{m} \in \mathbf{B}$, $\left(b_{i} b_{j}, b_{m}\right)=\left(b_{i}, \bar{b}_{j} b_{m}\right)$.

Let $(A, \mathbf{B})$ be a table algebra and $x \in A$. If $x=\alpha_{0} b_{0}+\alpha_{1} b_{1}+\cdots+\alpha_{k} b_{k}$, where $\alpha_{i} \in \mathbb{C}$, $0 \leq i \leq k$, then define $\operatorname{Supp}(x)=\left\{b_{i} \in \mathbf{B} \mid \alpha_{i} \neq 0\right\}$. An element $b_{i} \in \mathbf{B}$ is called linear if $\operatorname{Supp}\left(b_{i}^{n}\right)=\{1\}$ for some $n \in \mathbb{N}$. $(A, \mathbf{B})$ is called abelian if $b_{i}$ is linear for all $b_{i} \in \mathbf{B}$.

Let $\mathbf{C}$ be a subset of $\mathbf{B}$. If $\mathbf{C} \neq \emptyset$ and $\operatorname{Supp}\left(b_{i} b_{j}\right) \subseteq \mathbf{C}$ for all $b_{i}, b_{j} \in \mathbf{C}$, then $\mathbf{C}$ is called a table subset of $\mathbf{B}$. The table algebra $(A, \mathbf{B})$ is called simple if the only table subsets of $\mathbf{B}$ are $\mathbf{B}$ and $\{1\}$. For any $b \in \mathbf{B}$, let $\mathbf{B}_{b}=\cup_{i=1}^{\infty} \operatorname{Supp}\left(b^{i}\right)$. Then $\mathbf{B}_{b}$ is a table subset of $\mathbf{B}$. An element $b \in$ $\mathbf{B}$ is called faithful if $\mathbf{B}_{b}=\mathbf{B}$. Clearly $(A, \mathbf{B})$ is simple if and only if all $b_{i} \neq 1 \mathrm{in} \mathbf{B}$ are faithful.

The covering number $\operatorname{cn}(\mathbf{B})$ of a table algebra $(A, \mathbf{B})$ is the least positive integer $m$ such that $\operatorname{Supp}\left(b_{i}^{m}\right)=\mathbf{B}$ for all $b_{i} \neq 1$ in $\mathbf{B}$, if such $m$ exists, c.f. [1]. By [1, Theorem B], $c n(\mathbf{B})$ exists if and only if $(A, \mathbf{B})$ is simple and nonabelian, and $\operatorname{cn}(\mathbf{B}) \leq\left(|\mathbf{B}|^{2}-(r-1)^{2}\right) / 2$ if $\operatorname{cn}(\mathbf{B})$ exists, where $r$ is the number of real $b_{i} \neq 1$ in $\mathbf{B}$. In particular, if $(A, \mathbf{B})$ is a real table algebra and $c n(\mathbf{B})$ exists, then $c n(\mathbf{B}) \leq 2|\mathbf{B}|-2$. The next example presents an infinite family of real table algebras $(A, \mathbf{B})$ such that $c n(\mathbf{B})$ admits this upper bound.

Example 1.1. ([1] and [2]) Let $\mathbb{C}[\lambda]$ denote the ring of polynomials over $\mathbb{C}$ in the indeterminate $\lambda$. Let $p_{0}(\lambda), p_{1}(\lambda), \ldots, p_{k}(\lambda) \in \mathbb{C}[\lambda]$ be such that $p_{0}(\lambda)=1, p_{1}(\lambda)=\lambda$, and $p_{i}(\lambda)$ is a polynomial of degree $i(2 \leq i \leq k)$ defined by the recurrence:

$$
p_{i}(\lambda)=p_{1}(\lambda) p_{i-1}(\lambda)-p_{i-2}(\lambda), \quad i=2,3, \ldots, k
$$

Let $q(\lambda)=p_{1}(\lambda) p_{k}(\lambda)-p_{k-1}(\lambda)-p_{k}(\lambda),(q(\lambda))$ the ideal of $\mathbb{C}[\lambda]$ generated by $q(\lambda)$, $A=\mathbb{C}[\lambda] /(q(\lambda))$, the quotient ring of $\mathbb{C}[\lambda]$ with respect to $(q(\lambda))$, and $b_{i}=\bar{p}_{i}(\lambda) \in A$, $i=0,1, \ldots, k$. Then $(A, \mathbf{B})$ is a real table algebra with $\mathbf{B}=\left\{b_{0}, b_{1}, \ldots, b_{k}\right\}, c n(\mathbf{B})$ exists, and $\operatorname{cn}(\mathbf{B})=2|\mathbf{B}|-2$. Furthermore,

$$
\operatorname{Mat}\left(b_{1}\right)_{\mathbf{B}}=\left(\begin{array}{cccccc}
0 & 1 & & & & \\
1 & 0 & 1 & & & \\
& 1 & 0 & 1 & & \\
& & \ddots & \ddots & \ddots & \\
& & & 1 & 0 & 1 \\
& & & & 1 & 1
\end{array}\right)
$$

That is, the first intersection matrix of $(A, \mathbf{B})$ is of the form (2).

The next example provides a real table algebra whose first intersection matrix is of the form (2) while the covering number $\operatorname{cn}(\mathbf{B})$ does not exist.

Example 1.2. Let

$$
f_{0}(\lambda)=1, f_{1}(\lambda)=\lambda, f_{2}(\lambda)=\frac{1}{2} \lambda^{2}-4, f_{3}(\lambda)=\frac{1}{4} \lambda^{3}-3 \lambda, f_{4}(\lambda)=\frac{1}{8} \lambda^{4}-2 \lambda^{2}+4 .
$$

Let $f(\lambda)=\frac{1}{8} \lambda^{5}-\frac{1}{4} \lambda^{4}-\frac{5}{2} \lambda^{3}+4 \lambda^{2}+10 \lambda-8$, and $(f(\lambda))$ be the ideal of $\mathbb{C}[\lambda]$ generated by $f(\lambda)$. Let $A=\mathbb{C}[\lambda] /(f(\lambda))$, the quotient ring of $\mathbb{C}[\lambda]$ with respect to $(f(\lambda))$, and $b_{i}=$ $\bar{f}_{i}(\lambda) \in A, i=0,1,2,3,4$. Let $\mathbf{B}=\left\{b_{0}, b_{1}, b_{2}, b_{3}, b_{4}\right\}$. Then $\mathbf{B}$ is a basis of $\mathbf{A}$, and $b_{0}$ is the 包 Springer 
identity of A. Furthermore, we have that

$$
\begin{gathered}
b_{1}^{2}=8 b_{0}+2 b_{2}, b_{1} b_{2}=2 b_{1}+2 b_{3}, b_{1} b_{3}=2 b_{2}+2 b_{4}, b_{1} b_{4}=2 b_{3}+2 b_{4}, \\
b_{2}^{2}=8 b_{0}+2 b_{4}, b_{2} b_{3}=2 b_{1}+2 b_{4}, b_{2} b_{4}=2 b_{2}+2 b_{3}, \\
b_{3}^{2}=8 b_{0}+2 b_{3}, b_{3} b_{4}=2 b_{1}+2 b_{2}, b_{4}^{2}=8 b_{0}+2 b_{1} .
\end{gathered}
$$

So $(A, \mathbf{B})$ is a real table algebra, and

$$
\operatorname{Mat}\left(b_{1}\right)_{\mathbf{B}}=\left(\begin{array}{ccccc}
0 & 1 & & & \\
8 & 0 & 2 & & \\
& 2 & 0 & 2 & \\
& & 2 & 0 & 2 \\
& & & 2 & 2
\end{array}\right) .
$$

Since $\left\{b_{0}, b_{3}\right\}$ is a table subset, $(A, \mathbf{B})$ is not simple. Hence $c n(\mathbf{B})$ does not exist by [1, Theorem B].

\section{The Covering Number $c n(b)$ of $b \in \mathbf{B}$}

In this section we will first generalize the concept of the covering number of a table algebra $(A, \mathbf{B})$. For any $b \in \mathbf{B}$, we define the covering number $c n(b)$ of $b$, and present a necessary and sufficient condition for the existence of $c n(b)$, as well as an upper bound of $c n(b)$ when $c n(b)$ exists. These results generalize the results of Arad and Blau [1, Theorem B]. Then we will show that for a real $b \in \mathbf{B}, c n(b)$ exists and reaches the upper bound if and only if the intersection matrix of $b$ with respect to some reordering of $\mathbf{B}$ is tridiagonal of the form (2).

Definition 2.1. Let $(A, \mathbf{B})$ be a table algebra and $b \in \mathbf{B}$. If there exists $n \in \mathbb{N}$ such that $\operatorname{Supp}\left(b^{n}\right)=\mathbf{B}_{b}$, then the covering number of $\mathrm{b}$ is defined to be

$$
\operatorname{cn}(b):=\min \left\{n \in \mathbb{N} \mid \operatorname{Supp}\left(b^{n}\right)=\mathbf{B}_{b}\right\} .
$$

Let $(A, \mathbf{B})$ be a table algebra and $b \in \mathbf{B}$. If $\operatorname{Supp}\left(b^{n}\right)=\mathbf{B}_{b}$ for some $n \in \mathbb{N}$, then for all integers $m \geq n, \operatorname{Supp}\left(b^{m}\right)=\mathbf{B}_{b}$ by [1, Lemma 4.1(i)]. Furthermore, $c n(\mathbf{B})$ exists if and only if for any $b \neq 1$ in $\mathbf{B}, b$ is faithful and $c n(b)$ exists. Thus, if $c n(\mathbf{B})$ exists then

$$
c n(\mathbf{B})=\max \{c n(b) \mid b \in \mathbf{B} \backslash\{1\}\} .
$$

For any $b \in \mathbf{B}$ and any $n \in \mathbb{N}$, define

$$
\mathbf{B}_{b^{n}}=\bigcup_{i=1}^{\infty} \operatorname{Supp}\left(b^{n i}\right) .
$$

Clearly $\mathbf{B}_{b^{n}}$ is a table subset of $\mathbf{B}$. Recall that $b$ is linear if $\operatorname{Supp}\left(b^{n}\right)=\{1\}$ for some $n \in \mathbb{N}$. Thus $b$ is linear if and only if $\mathbf{B}_{b^{n}}=\{1\}$ for some $n \in \mathbb{N}$. The next proposition presents a necessary and sufficient condition for the existence of $c n(b)$.

Proposition 2.2. Let $(A, \mathbf{B})$ be a table algebra and $b \in \mathbf{B}$. Then $c n(b)$ exists if and only if for any $n \in \mathbb{N}, \mathbf{B}_{b^{n}}=\mathbf{B}_{b}$. 
Proof: If $c n(b)$ exists, then $\operatorname{Supp}\left(b^{m}\right)=\mathbf{B}_{b}$ for some $m \in \mathbb{N}$. So for any $n \in \mathbb{N}, \operatorname{Supp}\left(b^{n m}\right)=$ $\mathbf{B}_{b}$ by [1, Lemma 4.1(i)]. Therefore, $\mathbf{B}_{b^{n}}=\mathbf{B}_{b}$.

On the other hand, suppose for any $n \in \mathbb{N}, \mathbf{B}_{b^{n}}=\mathbf{B}_{b}$. Since $\mathbf{B}_{b}$ is a table subset, $1 \in \mathbf{B}_{b}$. Thus, $1 \in \operatorname{Supp}\left(b^{m}\right)$ for some $m \in \mathbb{N}$. Therefore, we have the following ascending chain

$$
\operatorname{Supp}\left(b^{m}\right) \subseteq \operatorname{Supp}\left(b^{2 m}\right) \subseteq \operatorname{Supp}\left(b^{3 m}\right) \subseteq \cdots
$$

But $\mathbf{B}_{b^{m}}=\mathbf{B}_{b}$. So there exists $l \in \mathbb{N}$ such that $\operatorname{Supp}\left(b^{l m}\right)=\mathbf{B}_{b}$. That is, $c n(b)$ exists.

From Proposition 2.2, we see that $\operatorname{cn}(\mathbf{B})$ exists if and only if any $b \neq 1$ in $\mathbf{B}$ is faithful and $c n(b)$ exists if and only if $(A, \mathbf{B})$ is simple and nonabelian.

The next proposition provides an upper bound for $c n(b)$ when $c n(b)$ exists.

Proposition 2.3. Let $(A, \mathbf{B})$ be a table algebra and $b \in \mathbf{B}$. Let $r$ be the number of real $b_{i} \neq 1$ in $\mathbf{B}_{b}$. If $c n(b)$ exists, then the following hold.

(i) $c n(\bar{b})$ exists, and $c n(\bar{b})=c n(b)$.

(ii) If $b$ is real, then $c n(b) \leq\left|\mathbf{B}_{b}\right|+r-1$. In particular, cn $(b) \leq 2\left|\mathbf{B}_{b}\right|-2$.

(iii) If $b$ is not real, then $c n(b) \leq\left(\left|\mathbf{B}_{b}\right|^{2}-(r-1)^{2}\right) / 2$.

Proof: (i) Since $\mathbf{B}_{b}$ is closed under ${ }^{-}$and $\mathbf{B}_{b}=\mathbf{B}_{\bar{b}}$, (i) holds.

(ii) Consider the ascending chain

$$
\left\{b_{0}\right\} \subset \operatorname{Supp}\left(b^{2}\right) \subseteq \operatorname{Supp}\left(b^{4}\right) \subseteq \operatorname{Supp}\left(b^{6}\right) \subseteq \cdots .
$$

Suppose there are $2 s$ nonreal elements in $\mathbf{B}_{b}$. Since $\operatorname{Supp}\left(b^{m}\right)$ is closed under ${ }^{-}$for any $m \in$ $\mathbb{N}$, we must have $\operatorname{Supp}\left(b^{2(s+r)}\right)=\operatorname{Supp}\left(b^{2(s+r)+2}\right)=\cdots$. But $c n(b)$ exists, $\operatorname{so} \operatorname{Supp}\left(b^{2(s+r)}\right)=$ $\mathbf{B}_{b}$. Hence, $c n(b) \leq 2(s+r)=\left|\mathbf{B}_{b}\right|+r-1$.

(iii) Assume that $\operatorname{cn}(b)=n$. Then $\operatorname{Supp}\left((b \bar{b})^{n}\right)=\operatorname{Supp}\left(b^{n} \bar{b}^{n}\right)=\mathbf{B}_{b}$. Consider the ascending chain

$$
\left\{b_{0}\right\} \subset \operatorname{Supp}(b \bar{b}) \subseteq \operatorname{Supp}\left((b \bar{b})^{2}\right) \subseteq \operatorname{Supp}\left((b \bar{b})^{3}\right) \subseteq \cdots
$$

As in the proof of (ii), we have that $\operatorname{Supp}\left((b \bar{b})^{s+r}\right)=\mathbf{B}_{b}$. By [1, Lemma 4.3], there is $m \leq$ $2 s+2$ such that $b_{0} \in \operatorname{Supp}\left(b^{m}\right)$. Hence $\operatorname{Supp}(b \bar{b}) \subseteq \operatorname{Supp}\left(b^{m}\right)$. Therefore, $\operatorname{Supp}\left(b^{m(s+r)}\right)=$ $\mathbf{B}_{b}$. So $c n(b) \leq m(s+r) \leq(2 s+2)(s+r)=\left(\left|\mathbf{B}_{b}\right|^{2}-(r-1)^{2}\right) / 2$.

As a direct consequence of Propositions 2.2 and 2.3, we have the following

Corollary 2.4. ([1, Theorem $\mathrm{B}])$ Let $(A, \mathbf{B})$ be a table algebra with $|\mathbf{B}|>1$. Then cn $(\mathbf{B})$ exists if and only if $(A, \mathbf{B})$ is simple and nonabelian. If cn $(\mathbf{B})$ exists, then cn $(\mathbf{B}) \leq\left(|\mathbf{B}|^{2}-\right.$ $\left.(r-1)^{2}\right) / 2$, where $r$ is the number of real $b_{i} \neq 1$ in $\mathbf{B}$.

The next theorem describes the intersection matrix of a real $b \in \mathbf{B}$ such that $c n(b)$ exists and reaches the upper bound. A similar result can be found in [7], but our proof here is different. We will need this theorem in next section.

Theorem 2.5. Let $(A, \mathbf{B})$ be a table algebra with $|\mathbf{B}|=k+1$, and $b \in \mathbf{B}$ be real. Then the following are equivalent. 
(i) $c n(b)$ exists and $c n(b)=2|\mathbf{B}|-2$.

(ii) $(A, \mathbf{B})$ is a real table algebra, and there is a reordering $\mathbf{B}^{\prime}$ of $\mathbf{B}$ such that

$$
\operatorname{Mat}(b)_{\mathbf{B}^{\prime}}=\left(\begin{array}{cccccc}
0 & 1 & & & & \\
\alpha_{0} & 0 & \gamma_{2} & & & \\
& \alpha_{1} & 0 & \gamma_{3} & & \\
& & \ddots & \ddots & \ddots & \\
& & & \alpha_{k-2} & 0 & \gamma_{k} \\
& & & & \alpha_{k-1} & \lambda_{k}
\end{array}\right) \text {, where } \alpha_{i}>0, \gamma_{j}>0, \lambda_{k}>0
$$

Proof: (ii) $\Rightarrow$ (i) We may assume that $\mathbf{B}^{\prime}=\mathbf{B}=\left\{b_{0}, b_{1}, \ldots, b_{k}\right\}$ and $b=b_{1}$. Then it is easy to show that $c n(b)$ exists and $c n(b)=2|\mathbf{B}|-2$.

(i) $\Rightarrow$ (ii) Since $c n(b) \leq 2\left|\mathbf{B}_{b}\right|-2$ by Proposition 2.3 , we see that $\left|\mathbf{B}_{b}\right|=|\mathbf{B}|$. So $b$ is faithful, and hence $\operatorname{Supp}\left(b^{2 k}\right)=\mathbf{B}$. From $c n(b)=2 k$ we get an ascending chain

$$
\{1\}=\operatorname{Supp}\left(b^{0}\right) \subset \operatorname{Supp}\left(b^{2}\right) \subset \operatorname{Supp}\left(b^{4}\right) \subset \cdots \subset \operatorname{Supp}\left(b^{2 k}\right)=\mathbf{B}
$$

such that $\operatorname{Supp}\left(b^{2 i}\right) \neq \operatorname{Supp}\left(b^{2 i+2}\right)$ for all $i=0,1,2, \ldots, k-1$. Hence

$$
\left|\operatorname{Supp}\left(b^{2 i+2}\right)\right|=\left|\operatorname{Supp}\left(b^{2 i}\right)\right|+1, \quad 0 \leq i \leq k-1 .
$$

Thus, all elements in any $\operatorname{Supp}\left(b^{2 i}\right)$ are real, $0 \leq i \leq k$. So $(A, \mathbf{B})$ is a real table algebra.

By (3) and (4), we may assume that

$$
\operatorname{Supp}\left(b^{2 i}\right)=\left\{b_{0}, b_{1}, \ldots, b_{i}\right\}, \quad 0 \leq i \leq k .
$$

Then $\operatorname{Supp}\left(b^{2}\right)=\left\{b_{0}, b_{1}\right\}$, and

$$
\operatorname{Supp}\left(b^{2 i+2}\right)=\operatorname{Supp}\left(b^{2 i}\right) \cup \operatorname{Supp}\left(b_{1} b_{i}\right), \quad 0 \leq i \leq k-1 .
$$

Thus,

$$
\left\{b_{i+1}\right\} \subseteq \operatorname{Supp}\left(b_{1} b_{i}\right) \subseteq\left\{b_{0}, b_{1}, \ldots, b_{i+1}\right\}, \quad 1 \leq i \leq k-1
$$

For any $i \in\{1,2, \ldots, k-1\}$, if $b_{l} \in \operatorname{Supp}\left(b_{1} b_{i}\right), 0 \leq l \leq i$, then $\left(b_{1} b_{i}, b_{l}\right) \neq 0$. Since $\mathbf{B}$ is real, we get $\left(b_{1} b_{l}, b_{i}\right) \neq 0$. So $i \leq l+1$ by (6). Hence $l \geq i-1$, and $\operatorname{Supp}\left(b_{1} b_{i}\right) \subseteq$ $\left\{b_{i-1}, b_{i}, b_{i+1}\right\}$. But $\left(b_{1} b_{i-1}, b_{i}\right) \neq 0$ by $(6)$, so $\left(b_{1} b_{i}, b_{i-1}\right) \neq 0$, and hence $b_{i-1} \in$ $\operatorname{Supp}\left(b_{1} b_{i}\right)$. Therefore, we get that

$$
\left\{b_{i-1}, b_{i+1}\right\} \subseteq \operatorname{Supp}\left(b_{1} b_{i}\right) \subseteq\left\{b_{i-1}, b_{i}, b_{i+1}\right\}, \quad 1 \leq i \leq k-1 .
$$

Similarly,

$$
\left\{b_{k-1}\right\} \subseteq \operatorname{Supp}\left(b_{1} b_{k}\right) \subseteq\left\{b_{k-1}, b_{k}\right\}
$$

On the other hand, we have an ascending chain

$$
\{b\}=\operatorname{Supp}(b) \subset \operatorname{Supp}\left(b^{3}\right) \subset \operatorname{Supp}\left(b^{5}\right) \subset \cdots \subset \operatorname{Supp}\left(b^{2 k+1}\right)=\mathbf{B}
$$


such that $\operatorname{Supp}\left(b^{2 i+1}\right) \neq \operatorname{Supp}\left(b^{2 i-1}\right)$ for all $i=1,2, \ldots, k$. Hence

$$
\left|\operatorname{Supp}\left(b^{2 i+1}\right)\right|=\left|\operatorname{Supp}\left(b^{2 i-1}\right)\right|+1, \quad 1 \leq i \leq k .
$$

In particular, $\left|\operatorname{Supp}\left(b^{3}\right)\right|=2$. Now we claim that

$$
b=b_{k} \text {. }
$$

If (9) is not true, then $b=b_{l}$ for some $0<l<k$. Hence by (7), $\left\{b_{l-1}, b_{l+1}\right\} \subseteq \operatorname{Supp}\left(b_{1} b_{l}\right) \subseteq$ $\operatorname{Supp}\left(b^{3}\right)$. But $b \in \operatorname{Supp}\left(b^{3}\right)$. So $\left|\operatorname{Supp}\left(b^{3}\right)\right| \geq 3$, a contradiction. Therefore, (9) holds. Hence,

$$
\operatorname{Supp}\left(b_{k}^{2}\right)=\left\{b_{0}, b_{1}\right\} \quad \text { and } \quad \operatorname{Supp}\left(b_{k}^{3}\right)=\left\{b_{k}, b_{k-1}\right\}
$$

More generally, we can prove that

$$
\operatorname{Supp}\left(b_{k}^{2 i+1}\right)=\left\{b_{k}, b_{k-1}, \ldots, b_{k-i}\right\}, \quad 0 \leq i \leq k .
$$

Now we show that

$$
\operatorname{Supp}\left(b_{k} b_{i}\right)=\left\{b_{k-i}, b_{k-i+1}\right\} \text { and } \operatorname{Supp}\left(b_{k} b_{k-i}\right)=\left\{b_{i}, b_{i+1}\right\}, \quad 1 \leq i<k
$$

by induction on $i$. First of all, $\operatorname{Supp}\left(b_{k} b_{1}\right)=\left\{b_{k-1}, b_{k}\right\}$ by (5), (8), and (9). Hence $b_{1} \in \operatorname{Supp}\left(b_{k} b_{k-1}\right)$. By (10), $\operatorname{Supp}\left(b_{k}^{4}\right)=\operatorname{Supp}\left(b_{k}^{2}\right) \cup \operatorname{Supp}\left(b_{k} b_{k-1}\right)$. Hence $\operatorname{Supp}\left(b_{k} b_{k-1}\right)=$ $\left\{b_{1}, b_{2}\right\}$ by (5). Thus, (11) holds for $i=1$. Now assume that $1 \leq l<k-1$ and (11) holds for all $i=1,2, \ldots, l$. Then we show that (11) holds for $i=l+1$. Since $\operatorname{Supp}\left(b_{k} b_{k-l}\right)=$ $\left\{b_{l}, b_{l+1}\right\}$ by induction, we see that $b_{k-l} \in \operatorname{Supp}\left(b_{k} b_{l+1}\right)$. But $\operatorname{Supp}\left(b_{k}^{2 l+1}\right)=\cup_{j=0}^{l} \operatorname{Supp}\left(b_{k} b_{j}\right)$ and $\operatorname{Supp}\left(b_{k}^{2 l+3}\right)=\cup_{j=0}^{l+1} \operatorname{Supp}\left(b_{k} b_{j}\right)$ by (5). So

$$
\left\{b_{k-l-1}, b_{k-l}\right\} \subseteq \operatorname{Supp}\left(b_{k} b_{l+1}\right) \subseteq\left\{b_{k}, b_{k-1}, \ldots, b_{k-l}, b_{k-l-1}\right\}
$$

For any $1 \leq j \leq l, b_{l+1} \notin \operatorname{Supp}\left(b_{k} b_{k-l+j}\right)$ by induction, so $b_{k-l+j} \notin \operatorname{Supp}\left(b_{k} b_{l+1}\right)$. Therefore, $\operatorname{Supp}\left(b_{k} b_{l+1}\right)=\left\{b_{k-l-1}, b_{k-l}\right\}$. Similary, we can prove that $\operatorname{Supp}\left(b_{k} b_{k-l-1}\right)=$ $\left\{b_{l+1}, b_{l+2}\right\}$. Hence (11) holds for $i=l+1$. Thus, (11) holds for all $1 \leq i<k$. Therefore, the intersection matrix of $b_{k}$ with respect to the reordering

$$
\mathbf{B}^{\prime}= \begin{cases}\left\{b_{0}, b_{k}, b_{1}, b_{k-1}, b_{2}, b_{k-2}, \ldots, b_{s-1}, b_{k-s+1}, b_{s}, b_{k-s}\right\}, & \text { if } k=2 s+1 \\ \left\{b_{0}, b_{k}, b_{1}, b_{k-1}, b_{2}, b_{k-2}, \ldots, b_{s-1}, b_{k-s+1}, b_{s}\right\}, & \text { if } k=2 s\end{cases}
$$

is a tridiagonal matrix of the form (2). So (ii) holds.

The next proposition provides a very simple sufficient condition under which $c n(\mathbf{B})$ exists for a real table algebra whose first intersection matrix is a tridiagonal matrix of the form (2). We will need this result in next section. 
Proposition 2.6. Let $(A, \mathbf{B})$ be a real table algebra such that $\mathbf{B}=\left\{b_{0}, b_{1}, \ldots, b_{k}\right\}$, and

$$
\operatorname{Mat}\left(b_{1}\right)_{\mathbf{B}}=\left(\begin{array}{cccccc}
0 & 1 & & & & \\
\alpha_{0} & 0 & \gamma_{2} & & & \\
& \alpha_{1} & 0 & \gamma_{3} & & \\
& & \ddots & \ddots & \ddots & \\
& & & \alpha_{k-2} & 0 & \gamma_{k} \\
& & & & \alpha_{k-1} & \lambda_{k}
\end{array}\right) \text {, where } \alpha_{i}>0, \gamma_{j}>0, \lambda_{k}>0
$$

If $2|\mathbf{B}|-1$ is a prime number, then $\operatorname{cn}(\mathbf{B})$ exists and $c n(\mathbf{B})=2|\mathbf{B}|-2$.

\section{Proof: Let}

$$
b_{k+1}=b_{k}, b_{k+2}=b_{k-1}, b_{k+3}=b_{k-2}, \ldots, b_{2 k}=b_{1}, b_{2 k+1}=b_{0} \text {. }
$$

Thus, for any $s, t \in\{0,1,2, \ldots, 2 k+1\}$,

$$
b_{r}=b_{s} \quad \text { if and only if } \quad r=s \text { or } r+s=2 k+1 .
$$

Since the first intersection matrix is of the form (2), by (12) we get that

$$
\operatorname{Supp}\left(b_{1} b_{i}\right)=\left\{b_{i-1}, b_{i+1}\right\}, \quad 1 \leq i \leq 2 k \text {. }
$$

Now we claim that for any $1 \leq r \leq i \leq k$,

$$
\left\{b_{i-r}, b_{i+r}\right\} \subseteq \operatorname{Supp}\left(b_{r} b_{i}\right) \subseteq\left\{b_{i-r}, b_{i-r+2}, b_{i-r+4}, \ldots, b_{i+r}\right\}
$$

We will prove (14) by induction on $r$. If $r=1$, (14) holds by (13). Now assume that $1 \leq$ $l<k$ and (14) holds for all $1 \leq r \leq l$. Since $b_{1} b_{l}=\alpha_{l-1} b_{l-1}+\gamma_{l+1} b_{l+1}$, for any $i$ such that $l+1 \leq i \leq k$, we have that

$$
b_{1} b_{l} b_{i}=\alpha_{l-1} b_{l-1} b_{i}+\gamma_{l+1} b_{l+1} b_{i} .
$$

Note that $b_{1} b_{l} b_{i}=b_{1}\left(b_{l} b_{i}\right)$, and $\left\{b_{i-l}, b_{i+l}\right\} \subseteq \operatorname{Supp}\left(b_{l} b_{i}\right)$ by induction. So $\left\{b_{i-l-1}\right.$, $\left.b_{i+l+1}\right\} \subset \operatorname{Supp}\left(b_{1} b_{l} b_{i}\right)$ by (13). Since

$$
\operatorname{Supp}\left(b_{l-1} b_{i}\right) \subseteq\left\{b_{i-l+1}, b_{i-l+3}, b_{i-l+5}, \ldots, b_{i+l-1}\right\}
$$

by induction, we see that $b_{i-l-1}, b_{i+l+1} \notin \operatorname{Supp}\left(b_{l-1} b_{i}\right)$ by (12). Therefore,

$$
\left\{b_{i-l-1}, b_{i+l+1}\right\} \subseteq \operatorname{Supp}\left(b_{l+1} b_{i}\right) .
$$

Furthermore, $\operatorname{since} \operatorname{Supp}\left(b_{l} b_{i}\right) \subseteq\left\{b_{i-l}, b_{i-l+2}, b_{i-l+4}, \ldots, b_{i+l}\right\}$ by induction, by (13) we get that $\operatorname{Supp}\left(b_{1} b_{l} b_{i}\right) \subseteq\left\{b_{i-l-1}, b_{i-l+1}, b_{i-l+3}, \ldots, b_{i+l+1}\right\}$. Thus, (15) implies that

$$
\operatorname{Supp}\left(b_{l+1} b_{i}\right) \subseteq\left\{b_{i-l-1}, b_{i-l+1}, b_{i-l+3}, \ldots, b_{i+l+1}\right\}
$$

So (14) holds for $r=l+1$. Therefore, (14) holds for all $1 \leq r \leq i \leq k$. 
For any $1 \leq r \leq k$, we will show that $b_{r}$ is faithful and nonabelian. First, (14) implies that $b_{0}, b_{2 r} \in \operatorname{Supp}\left(b_{r}^{2}\right)$. But $b_{0} \neq b_{2 r}$ by (12). So $b_{r}$ is not abelian. To prove that $b_{r}$ is faithful, we use induction on $r$. Clearly $b_{1}$ is faithful by (13). Now assume that $r>1$ and $b_{1}, b_{2}, \ldots, b_{r-1}$ are faithful. Then we prove that $b_{r}$ is faithful. Assume that $k \equiv l(\bmod r), 0 \leq l<r$. Then $b_{k-l} \in \mathbf{B}_{b_{r}}$ by (14). But $b_{k+l+1}=b_{k-l}$ by (12). So

$$
b_{k+l+1} \in \mathbf{B}_{b_{r}} .
$$

Note that $r>1$ and $2 k+1=2|\mathbf{B}|-1$ is a prime number. So $r \nmid(2 k+1)$. But $r \mid(k-l)$. Hence $r \nmid(k+l+1)$. Thus, we may assume that $k+l+1 \equiv s(\bmod r), 0<s<r$. Then $b_{s} \in \mathbf{B}_{b_{r}}$ by (14) and (16). But $b_{s}$ is faithful by induction assumption. So $\mathbf{B}_{b_{r}}=\mathbf{B}$, and hence $b_{r}$ is also faithful. Therefore, we have proved that $b_{1}, b_{2}, \ldots, b_{k}$ are all faithful.

Thus, $(A, \mathbf{B})$ is simple and nonabelian. Hence $\operatorname{cn}(\mathbf{B})$ exists by [1, Theorem B], and $\operatorname{cn}(\mathbf{B})=2|\mathbf{B}|-2$ by Theorem 2.5 .

Remark. Let $(A, \mathbf{B})$ be the table algebra in Example 1.2. Then $(A, \mathbf{B})$ is a real table algebra and $\operatorname{Mat}\left(b_{1}\right)_{\mathbf{B}}$ is of the form as in Proposition 2.6. But $(A, \mathbf{B})$ is not simple, so $\mathrm{cn}(\mathbf{B})$ does not exist. Note that $2|\mathbf{B}|-1=9$ is not a prime number. However, if $(A, \mathbf{B})$ is the table algebra in Example 1.1, then $|\mathbf{B}|=k+1, \operatorname{cn}(\mathbf{B})$ exists, and $\operatorname{cn}(\mathbf{B})=2|\mathbf{B}|-2$ for any positive integer $k$.

\section{Multiple P-polynomial structures}

In this section we will first present necessary and sufficient conditions under which for any $b \neq 1$ in a real table basis $\mathbf{B}, c n(b)$ exists and $c n(b)=2|\mathbf{B}|-2$. Then, using these results we prove that a standard real integral table algebra $(A, \mathbf{B})$ with $|\mathbf{B}| \geq 6$ is exactly isomorphic to the Bose-Mesner algebra of the association scheme of some ordinary $n$-gon with $n$ prime if and only if $(A, \mathbf{B})$ has a P-polynomial structure with respect to every $b \neq 1$ in $\mathbf{B}$, i.e. the intersection matrix of $b$ with respect to $\mathbf{B}$ or some reordering of $\mathbf{B}$ is tridiagonal of the form (1).

The next lemma is very useful.

Lemma 3.1. Let $(A, \mathbf{B})$ be a table algebra and $b \neq 1$ in $\mathbf{B}$ be real. Then $c n(b)$ exists and $c n(b)=2|\mathbf{B}|-2$ if and only if $b$ is faithful and $\left|\operatorname{Supp}\left(b b_{i}\right)\right|=2$ for any $b_{i} \neq 1$ in $\mathbf{B}$.

Proof: If $c n(b)$ exists and $c n(b)=2|\mathbf{B}|-2$, then by Theorem $2.5, b$ is faithful and $\left|\operatorname{Supp}\left(b b_{i}\right)\right|=2$ for any $b_{i} \neq 1$ in $\mathbf{B}$. On the other hand, suppose $b$ is faithful and $\left|\operatorname{Supp}\left(b b_{i}\right)\right|=$ 2 for any $b_{i} \neq 1$ in $\mathbf{B}$. We may assume that $b=b_{1}$. If $b_{1} \in \operatorname{Supp}\left(b_{1}^{2}\right)$, then $\mathbf{B}=\left\{b_{0}, b_{1}\right\}$. Hence, $c n(b)$ exists and $c n(b)=2=2|\mathbf{B}|-2$. If $b_{1} \notin \operatorname{Supp}\left(b_{1}^{2}\right)$, then we may assume that $\operatorname{Supp}\left(b_{1}^{2}\right)=\left\{b_{0}, b_{2}\right\}, b_{2} \neq b_{1}$. Since $b_{1}$ is real, $b_{2}$ is also real. So $\left(b_{1}^{2}, b_{2}\right) \neq 0$ implies that $\left(b_{1} b_{2}, b_{1}\right) \neq 0$. If $b_{2} \in \operatorname{Supp}\left(b_{1} b_{2}\right)$, then $\operatorname{Supp}\left(b_{1} b_{2}\right)=\left\{b_{1}, b_{2}\right\}$. Hence $\mathbf{B}=\left\{b_{0}, b_{1}, b_{2}\right\}$. So $c n(b)$ exists and $c n(b)=4=2|\mathbf{B}|-2$. If $b_{2} \notin \operatorname{Supp}\left(b_{1} b_{2}\right)$, then we may assume that $\operatorname{Supp}\left(b_{1} b_{2}\right)=\left\{b_{1}, b_{3}\right\}$. Since both $b_{1}$ and $b_{2}$ are real, $b_{3}$ is also real. More generally, we may assume that there are

$b_{0}, b_{1}, b_{2}, \ldots, b_{l} \in \mathbf{B}$, each $b_{i}$ is real, 
such that

$$
\operatorname{Supp}\left(b_{1} b_{i}\right)=\left\{b_{i-1}, b_{i+1}\right\}, \quad 1 \leq i<l .
$$

For any $1 \leq i<l-1,\left(b_{1} b_{i}, b_{l}\right)=0$. So $\left(b_{1} b_{l}, b_{i}\right)=0$, and hence $b_{i} \notin \operatorname{Supp}\left(b_{1} b_{l}\right), 1 \leq$ $i<l-1$. However, $b_{l-1} \in \operatorname{Supp}\left(b_{1} b_{l}\right)$. If $b_{l} \in \operatorname{Supp}\left(b_{1} b_{l}\right)$, since $b_{1}$ is faithful, we have $\mathbf{B}=$ $\left\{b_{0}, b_{1}, \ldots, b_{l}\right\}$, and the intersection matrix of $b_{1}$ with respect to $b_{0}, b_{1}, \ldots, b_{l}$ is tridiagonal of the form (2). So $c n(b)$ exists and $c n(b)=2|\mathbf{B}|-2$ by Theorem 2.5. If $b_{l} \notin \operatorname{Supp}\left(b_{1} b_{l}\right)$, then we may assume that $\operatorname{Supp}\left(b_{1} b_{l}\right)=\left\{b_{l-1}, b_{l+1}\right\}, b_{l+1} \neq b_{i}, 0 \leq i \leq l$, and $b_{l+1}$ is real. But $\mathbf{B}$ is a finite set and $b_{1}$ is faithful, we will finally get that $\mathbf{B}=\left\{b_{0}, b_{1}, \ldots, b_{k}\right\}$ and $\operatorname{Supp}\left(b_{1} b_{i}\right)=$ $\left\{b_{i-1}, b_{i+1}\right\}, 1 \leq i \leq k$, where $b_{k+1}=b_{k}$. So the intersection matrix of $b_{1}$ with respect to $b_{0}, b_{1}, \ldots, b_{k}$ is tridiagonal of the form (2). Hence, $c n(b)$ exists and $c n(b)=2|\mathbf{B}|-2$ by Theorem 2.5.

For a real table algebra $(A, \mathbf{B})$, the next proposition tells us when, for any $b \neq 1$ in $\mathbf{B}$, $c n(b)$ exists and $c n(b)=2|\mathbf{B}|-2$ in terms of the first intersection matrix.

Proposition 3.2. Let $(A, \mathbf{B})$ be a real table algebra such that $\mathbf{B}=\left\{b_{0}, b_{1}, \ldots, b_{k}\right\}$ with $k \geq 2$. Then the following are equivalent.

(i) For any $b \neq 1$ in $\mathbf{B}$, cn(b) exists and $c n(b)=2|\mathbf{B}|-2$.

(ii) $2|\mathbf{B}|-1$ is a prime number and, by a suitable renumbering of $b_{i} \in \mathbf{B}$ if necessary, the first intersection matrix is a tridiagonal matrix of the form (2):

$$
\left(\begin{array}{cccccc}
0 & 1 & & & & \\
\alpha_{0} & 0 & \gamma_{2} & & & \\
& \alpha_{1} & 0 & \gamma_{3} & & \\
& & \ddots & \ddots & \ddots & \\
& & & \alpha_{k-2} & 0 & \gamma_{k} \\
& & & & \alpha_{k-1} & \lambda_{k}
\end{array}\right) \text {, where } \alpha_{i}>0, \gamma_{j}>0, \lambda_{k}>0
$$

such that

$$
\frac{\alpha_{0}}{2}=\alpha_{1} \gamma_{2}=\alpha_{2} \gamma_{3}=\cdots=\alpha_{k-1} \gamma_{k}=\lambda_{k}^{2}, \quad \text { if } k>2,
$$

or

$$
\alpha_{1} \gamma_{2}+\lambda_{2}^{2}=\alpha_{0}, \quad \text { if } k=2
$$

Proof: Let

$$
\begin{aligned}
& b_{k+1}=b_{k}, b_{k+2}=b_{k-1}, b_{k+3}=b_{k-2}, \ldots, b_{2 k}=b_{1}, b_{2 k+1}=b_{0}, \\
& \gamma_{k+1}=\lambda_{k}, \gamma_{k+2}=\alpha_{k-1}, \gamma_{k+3}=\alpha_{k-2}, \ldots, \gamma_{2 k}=\alpha_{1}, \gamma_{2 k+1}=\alpha_{0},
\end{aligned}
$$

and

$$
\alpha_{k}=\lambda_{k}, \alpha_{k+1}=\gamma_{k}, \alpha_{k+2}=\gamma_{k-1}, \alpha_{k+3}=\gamma_{k-2}, \ldots, \alpha_{2 k-1}=\gamma_{2} .
$$


(i) $\Rightarrow$ (ii) By Theorem 2.5, we may assume that the first intersection matrix is of the form (2). Then we have

$$
b_{1} b_{i}=\alpha_{i-1} b_{i-1}+\gamma_{i+1} b_{i+1}, \quad 1 \leq i \leq 2 k .
$$

Thus, by $b_{1}^{2} b_{i}=b_{1}\left(b_{1} b_{i}\right)$, we get that

$$
b_{2} b_{i}=\frac{\alpha_{i-2} \alpha_{i-1}}{\gamma_{2}} b_{i-2}+\frac{\alpha_{i-1} \gamma_{i}+\alpha_{i} \gamma_{i+1}-\alpha_{0}}{\gamma_{2}} b_{i}+\frac{\gamma_{i+1} \gamma_{i+2}}{\gamma_{2}} b_{i+2}, \quad 2 \leq i \leq k .
$$

But for any $2 \leq i \leq k,\left|\operatorname{Supp}\left(b_{2} b_{i}\right)\right|=2$ by Lemma 3.1. So

$$
\alpha_{i-1} \gamma_{i}+\alpha_{i} \gamma_{i+1}-\alpha_{0}=0,2 \leq i \leq k
$$

In particular, (18) holds if $k=2$. Now assume that $k>2$. Then, from $\left(b_{1} b_{2}\right) b_{i}=b_{1}\left(b_{2} b_{i}\right)$, (19), (20), and (21) we get that

$$
\begin{aligned}
b_{3} b_{i}= & \frac{\alpha_{i-3} \alpha_{i-2} \alpha_{i-1}}{\gamma_{2} \gamma_{3}} b_{i-3}+\frac{\alpha_{i-2} \alpha_{i-1} \gamma_{i-1}-\alpha_{1} \alpha_{i-1} \gamma_{2}}{\gamma_{2} \gamma_{3}} b_{i-1} \\
& +\frac{\alpha_{i+1} \gamma_{i+1} \gamma_{i+2}-\alpha_{1} \gamma_{i+1} \gamma_{2}}{\gamma_{2} \gamma_{3}} b_{i+1}+\frac{\gamma_{i+1} \gamma_{i+2} \gamma_{i+3}}{\gamma_{2} \gamma_{3}} b_{i+3}, \quad 3 \leq i \leq k .
\end{aligned}
$$

But for any $3 \leq i \leq k,\left|\operatorname{Supp}\left(b_{3} b_{i}\right)\right|=2$ by Lemma 3.1. So

$$
\alpha_{i-2} \alpha_{i-1} \gamma_{i-1}-\alpha_{1} \alpha_{i-1} \gamma_{2}=0, \quad \text { and } \quad \alpha_{i+1} \gamma_{i+1} \gamma_{i+2}-\alpha_{1} \gamma_{i+1} \gamma_{2}=0, \quad 3 \leq i \leq k
$$

Therefore, (17) holds.

For any $1 \leq r \leq i \leq k,\left|\operatorname{Supp}\left(b_{r} b_{i}\right)\right|=2$ by Lemma 3.1. So Supp $\left(b_{r} b_{i}\right)=\left\{b_{i-r}, b_{i+r}\right\}$ by (14). If $2 k+1=2|\mathbf{B}|-1$ is not prime, then there are $r, s \in \mathbb{N}, 1<r, s<k$, such that $2 k+$ $1=r s$. Hence, $\left\{b_{0}, b_{r}, b_{2 r}, \ldots, b_{[(s-1) / 2] r}\right\}$ is a table subset of $\mathbf{B}$, a contradiction. Therefore, $2|\mathbf{B}|-1$ is a prime number, and (ii) holds.

(ii) $\Rightarrow$ (i) Note that $(A, \mathbf{B})$ is simple by Proposition 2.6. So By Lemma 3.1, it is enough to prove that

$$
\left|\operatorname{Supp}\left(b_{r} b_{i}\right)\right|=2, \quad 1 \leq r \leq i \leq k .
$$

Since the first intersection matrix is tridiagonal of the form (2), (22) is true if $r=1$. By induction on $r$, we can prove that

$$
b_{r} b_{i}=\frac{\alpha_{i-r} \alpha_{i-r+1} \cdots \alpha_{i-1}}{\gamma_{2} \gamma_{3} \cdots \gamma_{r}} b_{i-r}+\frac{\gamma_{i+1} \gamma_{i+2} \cdots \gamma_{i+r}}{\gamma_{2} \gamma_{3} \cdots \gamma_{r}} b_{i+r}, \quad 2 \leq r \leq i \leq k .
$$

So (22) is true for all $2 \leq r \leq k$. Hence (i) holds.

The next example provides an infinite family of real table algebras $(A, \mathbf{B})$ such that for any $b \neq 1$ in $\mathbf{B}, c n(b)$ exists and $c n(b)=2|\mathbf{B}|-2$.

Example 3.1. Let $k \in \mathbb{N}$ such that $k>1$ and $2 k+1$ is prime. Let $\mathbb{C}[\lambda]$ denote the ring of polynomials over $\mathbb{C}$ in the indeterminate $\lambda$. Let $p_{0}(\lambda), p_{1}(\lambda), \ldots, p_{k}(\lambda) \in \mathbb{C}[\lambda]$ be such that 包 Springer 
$p_{0}(\lambda)=1, p_{1}(\lambda)=\lambda, p_{2}(\lambda)=\frac{1}{2} \lambda^{2}-4$, and $p_{i}(\lambda)$ is a polynomial of degree $i(3 \leq i \leq k)$ defined by the recurrence:

$$
p_{i}(\lambda)=\frac{1}{2}\left[p_{1}(\lambda) p_{i-1}(\lambda)-2 p_{i-2}(\lambda)\right], \quad i=3,4, \ldots, k .
$$

Let $q(\lambda)=p_{1}(\lambda) p_{k}(\lambda)-2 p_{k-1}(\lambda)-2 p_{k}(\lambda),(q(\lambda))$ the ideal of $\mathbb{C}[\lambda]$ generated by $q(\lambda)$, and $A=\mathbb{C}[\lambda] /(q(\lambda))$, the quotient ring of $\mathbb{C}[\lambda]$ with respect to $(q(\lambda))$. Let $b_{i}=\bar{p}_{i}(\lambda) \in A$, $i=0,1, \ldots, k$, and $\mathbf{B}=\left\{b_{0}, b_{1}, b_{2}, \ldots, b_{k}\right\}$. Then $b_{0}=1$, the identity element of $\mathrm{A}, b_{1}^{2}=$ $8 b_{0}+2 b_{2}$, and

$$
b_{1} b_{i}= \begin{cases}2 b_{i-1}+2 b_{i+1}, & \text { if } 2 \leq i \leq k-1, \\ 2 b_{k-1}+2 b_{k}, & \text { if } i=k\end{cases}
$$

Furthermore, for any $2 \leq r \leq i \leq k$, we have

$$
b_{r} b_{i}= \begin{cases}2 b_{i-r}+2 b_{i+r}, & \text { if } i+r \leq k \\ 2 b_{i-r}+2 b_{2 k+1-i-r}, & \text { if } i+r>k\end{cases}
$$

Thus, $(A, \mathbf{B})$ is a real table algebra, and its first intersection matrix is a tridiagonal matrix of the form (2) as follows:

$$
\left(\begin{array}{cccccc}
0 & 1 & & & \\
8 & 0 & 2 & & & \\
& 2 & 0 & 2 & & \\
& & \ddots & \ddots & \ddots & \\
& & & 2 & 0 & 2 \\
& & & & 2 & 2
\end{array}\right)
$$

By Proposition 3.2, for any $b_{i} \neq 1$ in $\mathbf{B}, c n\left(b_{i}\right)$ exists and $c n\left(b_{i}\right)=2|\mathbf{B}|-2$.

The next theorem establishes a connection between multiple P-polynomial structures and covering numbers.

Theorem 3.3. Let $(A, \mathbf{B})$ be a real table algebra such that $|\mathbf{B}| \geq 6$. Then the following are equivalent.

(i) $(A, \mathbf{B})$ has a P-polynomial structure with respect to every $b \neq 1$ in $\mathbf{B}$, i.e. there is $a$ reordering $\mathbf{B}^{\prime}$ of $\mathbf{B}$ such that $\operatorname{Mat}(b)_{\mathbf{B}^{\prime}}$ is tridiagonal of the form (1).

(ii) For any $b \neq 1$ in $\mathbf{B}, c n(b)$ exists and $c n(b)=2|\mathbf{B}|-2$.

Proof: $(i) \Rightarrow(i i)$ We may assume that $\mathbf{B}=\left\{b_{0}=1, b_{1}, b_{2}, \ldots, b_{k}\right\}$ and

$$
\operatorname{Mat}\left(b_{1}\right)_{\mathbf{B}}=\left(\begin{array}{cccccc}
0 & 1 & & & & \\
\alpha_{0} & \beta_{1} & \gamma_{2} & & & \\
& \alpha_{1} & \beta_{2} & \gamma_{3} & & \\
& & \ddots & \ddots & \ddots & \\
& & & \alpha_{k-2} & \beta_{k-1} & \gamma_{k} \\
& & & & \alpha_{k-1} & \beta_{k}
\end{array}\right) \text {, where } \alpha_{i}>0, \gamma_{j}>0 \text {. }
$$


Then

$$
\begin{aligned}
b_{2} b_{i}= & \frac{1}{\gamma_{2}}\left[\alpha_{i-2} \alpha_{i-1} b_{i-2}+\left(\beta_{i-1}+\beta_{i}-\beta_{1}\right) \alpha_{i-1} b_{i-1}+\left(\alpha_{i-1} \gamma_{i}+\alpha_{i} \gamma_{i+1}-\alpha_{0}\right.\right. \\
& \left.\left.+\beta_{i}^{2}-\beta_{1} \beta_{i}\right) b_{i}+\left(\beta_{i}+\beta_{i+1}-\beta_{1}\right) \gamma_{i+1} b_{i+1}+\gamma_{i+1} \gamma_{i+2} b_{i+2}\right], \quad 2 \leq i \leq k-2 .
\end{aligned}
$$

So for any $2 \leq i \leq k-2, \quad b_{i-2}, b_{i+2} \in \operatorname{Supp}\left(b_{2} b_{i}\right)$. Since there is a reordering $\mathbf{B}^{\prime}$ of $\mathbf{B}$ such that $\operatorname{Mat}\left(b_{2}\right)_{\mathbf{B}^{\prime}}$ is tridiagonal of the form (1), we must have $\mathbf{B}^{\prime}=$ $\left\{b_{0}, b_{2}, b_{4}, b_{6}, \ldots, b_{5}, b_{3}, b_{1}\right\}$. Hence $b_{i-1}, b_{i+1} \notin \operatorname{Supp}\left(b_{2} b_{i}\right), 2 \leq i \leq k-2$. Therefore,

$$
\beta_{i-1}+\beta_{i}-\beta_{1}=0 \text { and } \beta_{i}+\beta_{i+1}-\beta_{1}=0, \quad 2 \leq i \leq k-2 .
$$

Thus,

$$
\beta_{1}=\beta_{3}=\beta_{5}=\ldots= \begin{cases}\beta_{k-2}, & \text { if } k \text { is odd } \\ \beta_{k-1}, & \text { if } k \text { is even, }\end{cases}
$$

and

$$
0=\beta_{2}=\beta_{4}=\ldots= \begin{cases}\beta_{k-1}, & \text { if } k \text { is odd } \\ \beta_{k-2}, & \text { if } k \text { is even }\end{cases}
$$

Hence,

$b_{2} b_{i}=\frac{1}{\gamma_{2}}\left[\alpha_{i-2} \alpha_{i-1} b_{i-2}+\left(\alpha_{i-1} \gamma_{i}+\alpha_{i} \gamma_{i+1}-\alpha_{0}\right) b_{i}+\gamma_{i+1} \gamma_{i+2} b_{i+2}\right], \quad 2 \leq i \leq k-2$.

Furthermore,

$b_{2} b_{k-1}=\frac{1}{\gamma_{2}}\left[\alpha_{k-3} \alpha_{k-2} b_{k-3}+\left(\alpha_{k-2} \gamma_{k-1}+\alpha_{k-1} \gamma_{k}-\alpha_{0}\right) b_{k-1}+\left(\beta_{k-1}+\beta_{k}-\beta_{1}\right) \gamma_{k} b_{k}\right]$,

and

$b_{2} b_{k}=\frac{1}{\gamma_{2}}\left[\alpha_{k-2} \alpha_{k-1} b_{k-2}+\left(\beta_{k-1}+\beta_{k}-\beta_{1}\right) \alpha_{k-1} b_{k-1}+\left(\alpha_{k-1} \gamma_{k}-\alpha_{0}+\beta_{k}^{2}-\beta_{1} \beta_{k}\right) b_{k}\right]$

So $b_{k} \in \operatorname{Supp}\left(b_{2} b_{k-1}\right)$ forces that

$$
\beta_{k} \neq \begin{cases}0, & \text { if } k \text { is even } \\ \beta_{1}, & \text { if } k \text { is odd. }\end{cases}
$$

Now we show that $\beta_{1}=0$. Note that

$$
\left(b_{1} b_{2}\right) b_{3}=\left(\alpha_{1} b_{1}+\gamma_{3} b_{3}\right) b_{3}=\alpha_{1}\left(\alpha_{2} b_{2}+\beta_{3} b_{3}+\gamma_{4} b_{4}\right)+\gamma_{3} b_{3}^{2} .
$$


First suppose that $k=5$. Then

$$
\begin{aligned}
b_{1}\left(b_{2} b_{3}\right)= & \frac{1}{\gamma_{2}}\left[\alpha_{1} \alpha_{2}\left(\alpha_{0} b_{0}+\beta_{1} b_{1}+\gamma_{2} b_{2}\right)+\left(\alpha_{2} \gamma_{3}+\alpha_{3} \gamma_{4}-\alpha_{0}\right)\left(\alpha_{2} b_{2}+\beta_{3} b_{3}+\gamma_{4} b_{4}\right)\right. \\
& \left.+\gamma_{4} \gamma_{5}\left(\alpha_{4} b_{4}+\beta_{5} b_{5}\right)\right] .
\end{aligned}
$$

If $\beta_{1} \neq 0$, then $b_{1} \in \operatorname{Supp}\left(b_{3}^{2}\right)$ by (27) and (28). But there is a reordering $\mathbf{B}^{\prime \prime}$ of $\mathbf{B}$ such that $\operatorname{Mat}\left(b_{3}\right)_{\mathbf{B}^{\prime \prime}}$ is tridiagonal of the form (1). Hence $b_{5} \notin \operatorname{Supp}\left(b_{3}^{2}\right)$. So $\beta_{5}=0$ by (27) and (28). Thus, the coefficient of $b_{4}$ in $b_{2} b_{5}$ is $-\beta_{1} \alpha_{4} / \gamma_{2}<0$ by (25), a contradiction. Therefore, we must have $\beta_{1}=0$ if $k=5$.

If $k>5$, then

$$
\begin{aligned}
b_{1}\left(b_{2} b_{3}\right)= & \frac{1}{\gamma_{2}}\left[\alpha_{1} \alpha_{2}\left(\alpha_{0} b_{0}+\beta_{1} b_{1}+\gamma_{2} b_{2}\right)+\left(\alpha_{2} \gamma_{3}+\alpha_{3} \gamma_{4}-\alpha_{0}\right)\left(\alpha_{2} b_{2}+\beta_{3} b_{3}+\gamma_{4} b_{4}\right)\right. \\
& \left.+\gamma_{4} \gamma_{5}\left(\alpha_{4} b_{4}+\beta_{5} b_{5}+\gamma_{6} b_{6}\right)\right] .
\end{aligned}
$$

So $b_{0}, b_{6} \in \operatorname{Supp}\left(b_{3}^{2}\right)$ by (27) and (29). But there is a reordering $\mathbf{B}^{\prime \prime}$ of $\mathbf{B}$ such that $\operatorname{Mat}\left(b_{3}\right)_{\mathbf{B}^{\prime \prime}}$ is tridiagonal of the form (1). So $b_{1} \notin \operatorname{Supp}\left(b_{3}^{2}\right)$. Hence $\beta_{1}=0$ by (27) and (29).

Therefore, we always have $\beta_{1}=0$. So from (23), (24), and (26) we see that

$$
\beta_{1}=\beta_{2}=\ldots=\beta_{k-1}=0 \text { but } \beta_{k} \neq 0
$$

Applying (30) to $\operatorname{Mat}\left(b_{2}\right)_{\mathbf{B}^{\prime}}$, we get that

$$
\alpha_{i-1} \gamma_{i}+\alpha_{i} \gamma_{i+1}-\alpha_{0}=0,2 \leq i \leq k-1, \quad \text { and } \quad \alpha_{k-1} \gamma_{k}+\beta_{k}^{2}-\alpha_{0}=0
$$

Since $b_{5} \in \operatorname{Supp}\left(b_{3}^{2}\right)$ (if $k=5$ ) or $b_{6} \in \operatorname{Supp}\left(b_{3}^{2}\right)$ (if $k>5$ ), we must have $b_{4} \notin \operatorname{Supp}\left(b_{3}^{2}\right)$. So (27), (28), and (29) imply that $\alpha_{1} \gamma_{2}=\alpha_{4} \gamma_{5}$. Therefore,

$$
\frac{\alpha_{0}}{2}=\alpha_{1} \gamma_{2}=\alpha_{2} \gamma_{3}=\cdots=\alpha_{k-1} \gamma_{k}=\beta_{k}^{2}
$$

Hence (ii) holds by Proposition 3.2.

(ii) $\Rightarrow$ (i) This follows directly from Theorem 2.5 .

Remark. It is clear that Theorem 3.3 is not true for $|\mathbf{B}|=3$. The following example shows that the theorem neither is true for $|\mathbf{B}|=4$. If $|\mathbf{B}|=5$, then $2|\mathbf{B}|-1=9$ is not prime, and hence Theorem 3.3(ii) does not hold by Theorem 3.2.

Example 3.2. Let

$$
f_{0}(\lambda)=1, f_{1}(\lambda)=\lambda, f_{2}(\lambda)=\lambda^{2}-3, f_{3}(\lambda)=\lambda^{3}-4 \lambda
$$

Let $f(\lambda)=\lambda^{4}-\lambda^{3}-6 \lambda^{2}+4 \lambda+6$, and $(f(\lambda))$ be the ideal of $\mathbb{C}[\lambda]$ generated by $f(\lambda)$. Let $A=\mathbb{C}[\lambda] /(f(\lambda))$, the quotient ring of $\mathbb{C}[\lambda]$ with respect to $(f(\lambda))$, and $b_{i}=\bar{f}_{i}(\lambda) \in A$, $i=0,1,2,3$. Let $\mathbf{B}=\left\{b_{0}, b_{1}, b_{2}, b_{3}\right\}$. Then $\mathbf{B}$ is a basis of $\mathrm{A}$, and $b_{0}$ is the identity of A. 
Furthermore, we have that

$$
\begin{gathered}
b_{1}^{2}=3 b_{0}+b_{2}, b_{1} b_{2}=b_{1}+b_{3}, b_{1} b_{3}=2 b_{2}+b_{3}, \\
b_{2}^{2}=3 b_{0}+b_{3}, b_{2} b_{3}=2 b_{1}+2 b_{2}, b_{3}^{2}=6 b_{0}+2 b_{1}+b_{3} .
\end{gathered}
$$

So $(A, \mathbf{B})$ is a real table algebra. Let $\mathbf{B}^{\prime}=\left\{b_{0}, b_{2}, b_{3}, b_{1}\right\}$ and $\mathbf{B}^{\prime \prime}=\left\{b_{0}, b_{3}, b_{1}, b_{2}\right\}$. Then

$$
\operatorname{Mat}\left(b_{1}\right)_{\mathbf{B}}=\left(\begin{array}{cccc}
0 & 1 & & \\
3 & 0 & 1 & \\
& 1 & 0 & 1 \\
& & 2 & 1
\end{array}\right), \operatorname{Mat}\left(b_{2}\right)_{\mathbf{B}^{\prime}}=\left(\begin{array}{llll}
0 & 1 & & \\
3 & 0 & 1 & \\
& 2 & 0 & 2 \\
& & 1 & 1
\end{array}\right), \operatorname{Mat}\left(b_{3}\right)_{\mathbf{B}^{\prime \prime}}=\left(\begin{array}{llll}
0 & 1 & & \\
6 & 1 & 2 & \\
& 1 & 0 & 2 \\
& & 2 & 2
\end{array}\right)
$$

That is, Theorem 3.3(i) holds for $(A, \mathbf{B})$. But $\operatorname{cn}\left(b_{3}\right)=3 \neq 2|\mathbf{B}|-2=6$. So Theorem 3.3(ii) does not hold for $(A, \mathbf{B})$.

Let $\mathcal{X}=\left(X,\left\{R_{i}\right\}_{0 \leq i \leq d}\right)$ be the association scheme of an ordinary $n$-gon. Let $A_{i}$ be the adjacency matrix with respect to $R_{i}$, and $\mathcal{A}$ the Bose-Mesner algebra of $\mathcal{X}$. Then $\left(\mathcal{A},\left\{A_{i}\right\}_{0 \leq i \leq d}\right)$ is a standard real integral table algebra such that its first intersection matrix is tridiagonal as follows:

$$
\left(\begin{array}{cccccc}
0 & 1 & & & & \\
2 & 0 & 1 & & & \\
& 1 & 0 & 1 & & \\
& & \ddots & \ddots & \ddots & \\
& & & 1 & 0 & 1 \\
& & & & 1 & 1
\end{array}\right) .
$$

Two table algebras $(A, \mathbf{B})$ and $(U, \mathbf{V})$ are called exactly isomorphic if there is an algebra isomorphism $\Phi: A \rightarrow U$ such that $\mathbf{V}=\{\Phi(b) \mid b \in \mathbf{B}\}$ and for any $b \in \mathbf{B}, b$ and $\Phi(b)$ have the same degree.

The next theorem is our main result. It answers the question proposed at the begining of the paper.

Theorem 3.4. Let (A, B) be a standard real integral table algebra such that $|\mathbf{B}| \geq 6$. Then the following are equivalent.

(i) (A, B) has a P-polynomial structure with respect to every $b \neq 1$ in $\mathbf{B}$, i.e. there is $a$ reordering $\mathbf{B}^{\prime}$ of $\mathbf{B}$ such that $\operatorname{Mat}(b)_{\mathbf{B}^{\prime}}$ is tridiagonal of the form (1).

(ii) For any $b \neq 1$ in $\mathbf{B}, c n(b)$ exists and $c n(b)=2|\mathbf{B}|-2$.

(iii) $2|\mathbf{B}|-1$ is a prime number, and $(A, \mathbf{B})$ is exactly isomorphic to the Bose-Mesner algebra of the association scheme of the ordinary $(2|\mathbf{B}|-1)$-gon.

Proof: (i) $\Rightarrow$ (ii) by Theorem 3.3. (iii) $\Rightarrow$ (i) is well-known. Now we show that (ii) $\Rightarrow$ (iii). By Proposition 3.2, 2|B $\mid-1$ is a prime number, and we may assume that the first intersection 을 Springer 
matrix of $(A, \mathbf{B})$ is a tridiagonal matrix of the form (2):

$$
\left(\begin{array}{cccccc}
0 & 1 & & & & \\
\alpha_{0} & 0 & \gamma_{2} & & & \\
& \alpha_{1} & 0 & \gamma_{3} & & \\
& & \ddots & \ddots & \ddots & \\
& & & \alpha_{k-2} & 0 & \gamma_{k} \\
& & & & \alpha_{k-1} & \lambda_{k}
\end{array}\right) \text {, where } \alpha_{i}>0, \gamma_{j}>0, \lambda_{k}>0 \text {, }
$$

such that $\alpha_{0} / 2=\alpha_{1} \gamma_{2}=\alpha_{2} \gamma_{3}=\cdots=\alpha_{k-1} \gamma_{k}=\lambda_{k}^{2}$. By [4, Proposition 5.8, p.96], $\alpha_{0}=$ $1+\alpha_{1}$. Thus $\alpha_{1}\left(2 \gamma_{2}-1\right)=1$. But all $\alpha_{i}$ and $\gamma_{j}$ are integers. So $\alpha_{0}=2$, and $\alpha_{1}=\alpha_{2}=\ldots=$ $\alpha_{k-1}=\gamma_{2}=\gamma_{3}=\ldots=\gamma_{k}=\lambda_{k}=1$. Therefore, $(A, \mathbf{B})$ and the Bose-Mesner algebra of the association scheme of the ordinary $(2|\mathbf{B}|-1)$-gon have the same first intersection matrix. Hence they are exactly isomorphic, and (iii) holds.

From the proof of Theorem 3.4, we have the following

Corollary 3.5. Let $(A, \mathbf{B})$ be a standard real integral table algebra such that $|\mathbf{B}|=4$. Then the following are equivalent.

(i) For any $b \neq 1$ in $\mathbf{B}, c n(b)$ exists and $c n(b)=2|\mathbf{B}|-2$.

(ii) (A, B) is exactly isomorphic to the Bose-Mesner algebra of the association scheme of the ordinary 7-gon.

Acknowledgments The author would like to thank the referees; their comments improved the exposition of the paper.

\section{References}

1. Z. Arad and H. Blau, On table algebras and applications to finite group theory, J. Algebra 138 (1991), 137-185.

2. Z. Arad and H. Blau, An infinite family of nonabelian simple table algebras not induced by finite nonabelian simple groups, in "Groups St. Andrews 1989," Vol. 1, pp. 29-37, Cambridge Univ. Press, Cambridge, UK, 1991.

3. A. Brouwer, A. Cohen, and A. Neumaier, Distance-Regular Graphs, Springer-Verlag Berlin Heidelberg, 1989.

4. E. Bannai and T. Ito, "Algebraic Combinatorics I: Association Schemes," Benjamin/Cummings, Menlo Park, CA, 1984.

5. H. I. Blau, Quotient structures in C-algebras, J. Algebra 177 (1995), 297-337.

6. H. Suzuki, A note on association schemes with two P-polynomial structures of type III, J. Combin. Theory Ser. A 74 (1996), 158-168.

7. B. Xu, Polynomial table algebras and their covering numbers, J. Algebra 176 (1995), 504-527. 